\title{
Diagnóstico do uso de ferramentas de gestão por proprietários rurais de Uberlândia - MG.
}

\section{Diagnostic of use of management tools by rural owners from Uberlândia -MG.}

\author{
Fernando Caixeta Lisboa ${ }^{1}$, Matheus Henrique Medeiros ${ }^{2}$, Jéssica Laura Miranda Peixoto ${ }^{2}$, Leôncio Campos Gouveia ${ }^{2}$, \\ Washington Ribeiro do Nascimento ${ }^{2}$
}

Resumo: O produtor rural deve se adequar, na tentativa de tornar-se consciente da gestão de sua propriedade rural, pois um melhor gerenciamento resulta em maior lucratividade e em menor custo de produção. Sendo assim, o objetivo deste trabalho foi realizar um diagnóstico do uso de ferramentas de gestão por produtores rurais do município de Uberlândia - MG. Para tanto, realizou-se a aplicação de formulários estruturados a 23 produtores rurais da referida localidade, através de pesquisa de campo, obtendo-se assim, panoramas quanto ao uso destas ferramentas. Os resultados indicam a necessidade de aprimoramento e do uso das ferramentas de gestão utilizada pelos proprietários rurais entrevistados. Detectou-se, ainda, a necessidade de abertura de um curso para esclarecimento do uso dessas ferramentas.

Palavras-chaves: Diagnóstico; ferramentas; gestão; produtores rurais.

\begin{abstract}
The farmer should suit in an attempt to become aware of the management in his farm, for better management results in higher profitability and lower production cost. Thus, this research aimed to make a diagnosis of the use of management tools by farmers in Uberlândia - MG city. Therefore, we carried out the application forms structured to 23 farmers of that locality, through field research, thus obtaining, panoramas on the use of these tools. The results indicate the need for improvement and the use of management tools used by respondent's landowners. It turned out, though, the need for opening a course for clarification of the use of these tools.
\end{abstract}

Key words: Diagnosis; tools; management; farmes.

\footnotetext{
*Autor para correspondência

Recebido para publicação em 19/02/2015; aprovado em 30/05/2015

${ }^{1}$ Mestre Profissional em Inovação Tecnológica pela Universidade Federal do Triângulo Mineiro (UFTM), com pesquisa na área de processos tecnológicos envolvendo o desenvolvimento de microalgas. Atualmente exerce atividades como Professor Efetivo do Instituto Federal de Educação, Ciência e Tecnologia do Triângulo Mineiro - Campus Uberlândia. E-mail: fernandocaixeta@iftm.edu.br

${ }^{2}$ Alunos do curso técnico em Agropecuária do Instituto Federal de Educação, Ciência e Tecnologia do Triângulo Mineiro - Campus Uberlândia.
} 


\section{INTRODUÇÃO}

Sob o cenário atual do rápido crescimento da população humana, a eficiência e produtividade das empresas rurais são essenciais para a manutenção da sustentabilidade da organização, que se apresenta como um desafio global (TSCHARNTKE et al., 2012).

O Brasil, reconhecidamente um país de base fortemente agroindustrial, cresceu sua participação no comércio mundial nas últimas décadas intensificando seus sistemas agroindustriais. Deste total de produção agropecuária cerca de $60 \%$ correspondem a propriedades familiares, com baixo nível de conhecimento de ferramentas administrativas, importantes para melhorar a gestão da propriedade (COSTA, 2012).

Neste sentido, procura-se melhorar a administração dos recursos disponíveis e potenciais visando o melhor uso da terra, água, máquinas e equipamentos e pessoal em uma propriedade rural (NAGAOKA et al., 2011). Profissionalizalas com base nas ciências administrativas é, portanto, necessário, pois esta ação faz com que os procedimentos fiquem mais claros e mais eficazes.

Fazem-se pertinentes algumas estratégias para prover conhecimento aos produtores e trabalhadores da zona rural sobre as atualizações administrativas, sendo assim, o gestor pode contribuir para a melhoria da qualidade de vida, atuando como agente colaborador e impulsionador de reformas no processo de inovação da propriedade (MARTINS et al., 2014).

O surgimento de novas demandas de mercado e a percepção de oportunidades de geração de emprego e renda tem mostrado a urgência na utilização de ferramentas de gestão. Sendo assim, esse uso contribuirá para a estabilidade por meio de decisões viabilizadoras de benefícios e minimização das incertezas e variações abruptas no que diz respeito à gestão das organizações.

Baixa disponibilidade de capital, baixa expectativa de renda, baixo grau de educação formal, a dependência das assistências técnica, o número elevado de propriedades e a instabilidade econômica do país são razões que fazem Minas Gerais carecer de melhorias no que tange a administração das propriedades, que caracteriza grande parte dos produtores (GODINHO et al., 2013).

Princípios de finanças, marketing, recursos humanos e operações devem ser adotadas por estes proprietários para que as atividades tenham o menor impacto de custos e o maior impacto de produtividade e consequentemente lucratividade.

Dessa forma, elabora-se o questionamento da pesquisa pautado no seguinte problema de investigação: Estão sendo utilizadas ferramentas de gestão pelos proprietários rurais no município de Uberlândia - Minas Gerais?

Portanto, o objetivo deste trabalho foi realizar um diagnóstico do uso de ferramentas de gestão por produtores rurais do município de Uberlândia - MG, expondo características administrativas em um questionário estruturado respondido por 23 produtores.

Nas seções seguintes expõe-se os procedimentos metodológicos utilizados nesta pesquisa. Evidencia-se os resultados e discussões acerca do tema e, por fim, conclui o trabalho com detalhes dos objetivos e resultados alcançados.

\section{MATERIAL E MÉTODOS}

Nas Ciências Sociais, há dois caminhos que podem ser percorridos na construção da pesquisa científica: o quantitativo e o qualitativo. A diferença básica entre eles é que os métodos quantitativos produzem resultados mensuráveis em termos de quantidade, intensidade e frequência, enquanto os métodos qualitativos buscam a natureza social da construção da realidade e o modo pelo qual a experiência social surge e adquire significado (MINAYO, 1994).

Em razão de a natureza deste trabalho realizar um estudo junto aos proprietários rurais, optou-se pela metodologia de pesquisa qualitativa, já que é a que melhor consegue captar esse universo dos significados à luz do entendimento dos atores envolvidos.

O presente trabalho foi realizado junto a produtores rurais do munícipio de Uberlândia, em Minas Gerais. Este município possui uma área total de 411.582 hectares, dos quais 280.000 são de áreas agricultáveis. Deste total, $70 \mathrm{mil}$ hectares são ocupados com exploração agrícola e 127 mil com exploração pecuária. As pequenas e médias propriedades (áreas com até 100 hectares) representam $68 \%$ da totalidade existente no município e têm sido o foco de diversas políticas públicas para o aumento de sua produção e consequentemente de sua lucratividade (VASCONCELOS et al., 2011).

Para a coleta dos dados foi utilizada a aplicação de um questionário composto por questões objetivas com respostas de múltipla escolha. Foi estruturado em duas partes: informações socioeconômicas e informações sobre a utilização de ferramentas de gestão. Os questionários foram aplicados entre os meses de março a abril de 2014, junto a 23 proprietários rurais do munícipio de Uberlândia.

A escolha dos proprietários rurais deu-se de forma não probabilística por acessibilidade (quando o pesquisador tem acesso ao objeto de análise que permita continuar seus estudos).

A análise dos dados foi realizada nas duas etapas separadas do questionário. Na primeira etapa são descritas as características socioeconômicas das propriedades rurais e em uma segunda etapa do uso de ferramentas de gestão. Os dados coletados foram analisados por meio de estatística descritiva e distribuição de frequência, conforme verificados a seguir.

\section{RESULTADOS E DISCUSSÃO}

\section{Caracterização socioeconômicas das propriedades rurais.}

Nesta seção pretende-se conhecer o perfil socioeconômico do proprietário rural estudado.

Dos proprietários rurais pesquisados, constata-se que $69,57 \%$ dos entrevistados estão na faixa etária acima dos 40 anos o que pode ser visto na tabela 1 .

Tabela 1 - Faixa etária dos proprietários entrevistados

\begin{tabular}{lcc}
\hline & Proprietários rurais & Percentuais $(\%)$ \\
\hline Até 20 anos & $\mathbf{2}$ & $\mathbf{4 , 3 5}$ \\
21 a 25 anos & $\mathbf{1}$ & $\mathbf{4 , 3 5}$ \\
26 a 30 anos & $\mathbf{1}$ & $\mathbf{8 , 7 0}$ \\
31 a 35 anos & $\mathbf{2}$ & $\mathbf{4 , 3 5}$ \\
36 a 40 anos & $\mathbf{1}$ & $\mathbf{8 , 7 0}$ \\
Mais de 40 anos & $\mathbf{1 6}$ & $\mathbf{6 9 , 5 7}$ \\
\hline
\end{tabular}

Fonte: Elaborado pelos autores. 
Nota-se tendência de envelhecimento da população entrevistada. Sabe-se que a população brasileira está em processo de envelhecimento. Conforme os primeiros resultados do XII Recenseamento Geral do Brasil do Censo 2010, realizado pelo Instituto Brasileiro de Geografia e Estatística (IBGE), a proporção de idosos (acima de 65 anos) aumentou de 5,9\% em 2000 para 7,4\% em 2010 (IBGE, 2010).

Resultados semelhantes foram encontrados por Costa et al. (2014) que realizaram diagnóstico rural com 23 agricultores familiares, encontrando-os com idade média por volta de 50 anos.

Todos os produtores entrevistados são alfabetizados, entretanto, $8,70 \%$ tem o nível de ensino básico, $21,74 \%$ estão no nível de ensino fundamental, $30,43 \%$ estão com nível de ensino médio, $21,74 \%$ estão com nível superior completo e 17, 39\% possuem algo curso em nível de pós-graduação, conforme pode ser visto na tabela 2 .

Tabela 2 - Escolaridade dos proprietários entrevistados Proprietários rurais Percentuais (\%)

\begin{tabular}{llc}
\hline Ensino Básico & $\mathbf{2}$ & $\mathbf{8 , 7 0}$ \\
Ensino Fundamental & $\mathbf{5}$ & $\mathbf{2 1 , 7 4}$ \\
Ensino Médio & $\mathbf{7}$ & $\mathbf{3 0 , 4 3}$ \\
Superior Completo & $\mathbf{4}$ & $\mathbf{1 7 , 3 9}$ \\
Pós - Graduação & $\mathbf{5}$ & $\mathbf{2 1 , 7 4}$ \\
\hline
\end{tabular}

Fonte: Elaborado pelos autores.

O nível de escolaridade do produtor influencia seu interesse na busca de novos conhecimentos, na medida em que possibilita estabelecer comportamentos mais desejáveis na assimilação e adoção das informações recebidas (SILVA, 1995).

Podemos perceber que a maioria dos produtores que responderam a esta pesquisa são pequenos ou médios produtores rurais já que $73,91 \%$ dos produtores entrevistados afirmaram que sua propriedade rural tem menos que 100 hectares, conforme pode ser visualizado na tabela 3 .

Tabela 3 - Tamanho da propriedade rural em hectares

\begin{tabular}{lcc}
\hline & Proprietários rurais & Percentuais $(\%)$ \\
\hline Até 25 hectares. & $\mathbf{8}$ & $\mathbf{3 4 , 7 8}$ \\
Entre 26 e 50 hectares. & $\mathbf{5}$ & $\mathbf{2 1 , 7 4}$ \\
Entre 51 e 60 hectares. & $\mathbf{2}$ & $\mathbf{8 , 7 0}$ \\
Entre 61 e 100 hectares & $\mathbf{2}$ & $\mathbf{8 , 7 0}$ \\
Acima de 100 hectares & $\mathbf{6}$ & $\mathbf{2 6 , 0 9}$ \\
\hline Fonte: Elaborado pelos autores &
\end{tabular}

Fonte: Elaborado pelos autores.

Segundo a Constituição Federal brasileira, materializada na Lei $\mathrm{n}^{\mathrm{o}} 11.326$ de julho de 2006, considera-se agricultor familiar, dentre outros aspectos, aquele que desenvolve atividades econômicas no meio rural e que não possui propriedade rural maior que 4 módulos fiscais, que no município de Uberlândia é 20 hectares (BRASIL, 2006).

Dentre as principais atividades desenvolvidas os dados indicam que $43,48 \%$ dos produtores rurais afirmaram que sua principal atividade e a ordenha de leite conforme pode ser visto na tabela 4 .
Tabela 4 - Principal atividade da propriedade rural

\begin{tabular}{lcc}
\hline & Proprietários rurais & Percentuais $(\%)$ \\
\hline Cana-de-açúcar & $\mathbf{1}$ & $\mathbf{4 , 3 5}$ \\
Gado de Corte & $\mathbf{8}$ & $\mathbf{3 4 . 7 8}$ \\
Frutas e Hortaliças & $\mathbf{2}$ & $\mathbf{8 , 7 0}$ \\
Leite & $\mathbf{1 0}$ & $\mathbf{4 3 , 4 8}$ \\
Piscicultura & 1 & $\mathbf{4 , 3 5}$ \\
Suinocultura & $\mathbf{1}$ & $\mathbf{4 , 3 5}$ \\
\hline
\end{tabular}

Fonte: Elaborado pelos autores.

O Brasil é um dos maiores produtores mundiais de leite, ocupando o $6^{\circ}$ lugar no ranking em 1996, com 19,84 bilhões de litros ou 4,2\% da produção total (SEBRAE-MG, 1997). A região sudeste é a maior produtora de leite do país. Conforme dados de Revista Gleba (1997), a região é responsável por $45,1 \%$ da produção brasileira, destacando-se o estado de Minas Gerais, com 29,1\% da produção nacional.

Foi possível perceber que 22 dos 23 proprietários afirmaram haver de 1 a 5 funcionários em sua propriedade, conforme pode ser visualizado na tabela 5 .

Tabela 5 - Quantidade de trabalhadores na propriedade rural

\begin{tabular}{lcc}
\hline & Proprietários rurais & Percentuais $(\%)$ \\
\hline De 1 a 5 & $\mathbf{2 2}$ & $\mathbf{9 5 , 6 5}$ \\
De 6 a 10 & $\mathbf{1}$ & $\mathbf{4 , 3 5}$ \\
\hline Fonte: Elaborado pelos autores. &
\end{tabular}

Houveram relatos de alguns proprietários de que os funcionários seriam os próprios membros da família, o que também segundo a lei $\mathrm{n}^{\mathrm{o}} 11.326$ de julho de 2006, pode caracterizar a agricultura familiar (BRASIL, 2006).

\section{Diagnóstico de utilização das ferramentas de gestão.}

Nesta seção pretende-se conhecer se o proprietário rural estudado, utiliza as ferramentas de gestão.

\section{Recursos humanos.}

O resultado sobre do diagnóstico de valorização do funcionário pode ser visualizado na tabela 6 .

Tabela 6 - Realização de algum tipo de valorização do funcionário na propriedade rural

\begin{tabular}{lcc} 
& Proprietários rurais & Percentuais $(\%)$ \\
\hline Sim & $\mathbf{8}$ & $\mathbf{6 5 , 2 2}$ \\
Não & $\mathbf{1 5}$ & $\mathbf{3 4 , 7 8}$ \\
\hline
\end{tabular}

Fonte: Elaborado pelos autores.

Os resultados indicaram que $65,22 \%$ dos entrevistados afirmaram não fazer nenhum tipo de valorização do funcionário. Ferramentas de gestão estratégica de recursos humanos são amplamente utilizadas como forma de captação, valorização e retenção do capital intelectual nas corporações, tornando-as capazes de enfrentar as adversidades do mercado, dentre elas destaca-se a valorização do funcionário (CAMPOS et al., 2014).

Os recursos organizacionais e humanos nas propriedades rurais, por vezes, são difíceis de ser separados, afinal muitas das ações organizacionais são humanas e estão diretamente relacionadas à postura dos empresários nas organizações e da política organizacional para com os funcionários (CARVALHO et al., 2014). 


\section{Finanças}

Os resultados da realização de controle financeiro podem ser observados na tabela 7 .

Tabela 7 - Realização de controle financeiro da propriedade rural

\begin{tabular}{lcc}
\hline & $\begin{array}{c}\text { Proprietários } \\
\text { rurais }\end{array}$ & Percentuais (\%) \\
\hline Não realizo. & $\mathbf{6}$ & $\mathbf{2 6 , 0 9}$ \\
Raramente. & $\mathbf{8}$ & $\mathbf{1 7 , 3 9}$ \\
Realizo com frequência. & $\mathbf{2}$ & $\mathbf{1 7 , 3 9}$ \\
Sempre realizo. & $\mathbf{1 0}$ & $\mathbf{3 9 , 1 3}$ \\
\hline
\end{tabular}

Fonte: Elaborado pelos autores.

Como pode ser observado, a maioria $39,13 \%$ afirmaram que realizam o controle financeiro. Entretanto, um número significativo de proprietários $(26,09 \%)$ afirmou não o realizar.

No caso da atividade rural, a análise dos custos permite ao produtor conhecer os resultados financeiros de sua empresa. A ajudando-o a tomar decisões corretas e a encarar seu sistema de produção como uma empresa (GARCIA; FERNANDES, 2014).

Pinto \& Franco (2014) analisaram os custos de produção na terminação do gado de corte em regime de confinamento. Como resultado obteve-se que a atividade mostrou-se rentável e a análise financeira demonstrou importância para que o produtor rural continuasse a investir nesta atividade.

Os resultados sobre o fato de possuir a assistência de um contador podem ser observados na tabela 8 .

Tabela 8 - Possui a assistência de um contador

\begin{tabular}{lcc}
\hline & Proprietários rurais & Percentuais $(\%)$ \\
\hline Sim. & $\mathbf{9}$ & $\mathbf{6 0 , 8 7}$ \\
Não. & $\mathbf{1 4}$ & $\mathbf{3 9 , 1 3}$ \\
\hline
\end{tabular}

Fonte: Elaborado pelos autores.

A maioria dos entrevistados $(60,87 \%)$ afirmaram que não possuem a assistência de um contador. No momento da tomada de decisão é papel da contabilidade o fornecimento de informações relevantes que orientem o administrador ou gerente para as melhores decisões. Portanto, a falta desse profissional pode acarretar em problemas de interpretação e análise de dados para a tomada de decisão.

Magro et al. (2014) verificaram a aplicabilidade da contabilidade rural e de custos para a melhoria da gestão da atividade leiteira em uma propriedade rural localizada no Oeste de Santa Catarina. Como resultados obteve-se que a contabilidade rural e de custos são técnicas conjuntas, que se utilizadas de maneira correta, possibilitam resultados importantes para a melhoria na gestão visando à eficiência da atividade.

\section{Produção}

Os resultados sobre realização de controle de produtividade da propriedade rural, pode ser visualizado na tabela 9 .
Tabela 9 - Realiza controle de produtividade na propriedade rural

\begin{tabular}{lcc}
\hline & $\begin{array}{c}\text { Proprietários } \\
\text { rurais }\end{array}$ & Percentuais $(\%)$ \\
\hline Não realizo. & $\mathbf{3}$ & $\mathbf{1 3 , 0 4}$ \\
Raramente. & $\mathbf{4}$ & $\mathbf{1 7 , 3 9}$ \\
Realizo com frequência. & $\mathbf{6}$ & $\mathbf{2 6 , 0 9}$ \\
Sempre realizo. & $\mathbf{1 0}$ & $\mathbf{4 3 , 4 8}$ \\
\hline
\end{tabular}

Fonte: Elaborado pelos autores.

A maioria dos entrevistados afirmaram que sempre realizam este controle. A produtividade da atividade agrícola desemprenha papel fundamental para que o proprietário saiba a real situação e possa tomar medidas diante disso.

Resultado semelhante foi encontrado por Fontes et al. (2013) avaliaram a percepção de agricultores familiares quanto a eficiência do seu estilo de produção e os entraves a sua adoção no território Sul Sergipano. Como resultado obtiveram que o estilo de produção de agroflorestal sucessional se adéqua a realidade de produção local e que este foi capaz de responder as expectativas técnicas e de produtividade dos entrevistados.

Embora a maioria dos produtores afirmaram realizar este controle é possível que haja engano quanto ao que é esse controle. Godinho et al. (2013) constataram em pesquisa realizada com produtores rurais da microrregião de São João Batista do Glória (MG) que os proprietários rurais não costumam realizar o controle diariamente limitando-se a uma expectativa de produção advinda do total de venda em um mesmo período já analisado.

\section{Comercialização na propriedade rural.}

Os resultados sobre a forma que é feita a comercialização dos produtos agrícolas podem ser visualizados na tabela 10 .

Tabela 10 - Formas de comercialização na propriedade rural

Proprietários Percentuais

\begin{tabular}{lcc} 
& $\begin{array}{c}\text { Proprietários } \\
\text { rurais }\end{array}$ & $\begin{array}{c}\text { Percentuais } \\
(\%)\end{array}$ \\
\hline Cooperativas. & $\mathbf{8}$ & $\mathbf{3 4 , 7 8}$ \\
Direto ao consumidor. & $\mathbf{5}$ & $\mathbf{2 1 , 7 4}$ \\
Frigorífico & $\mathbf{5}$ & $\mathbf{2 1 , 7 4}$ \\
Indústria & $\mathbf{1}$ & $\mathbf{4 , 3 5}$ \\
Uso de intermediário de & $\mathbf{3}$ & \\
vendas & & $\mathbf{1 3 , 0 4}$ \\
Leilão & $\mathbf{1}$ & $\mathbf{4 , 3 5}$ \\
\hline
\end{tabular}

Fonte: Elaborado pelos autores.

A maioria dos entrevistados respondeu que realiza a comercialização por cooperativas $(34,78 \%)$. Como a maioria dos produtores respondeu produzir leite, é normal que se realize a utilização do cooperativismo.

Sabe que a eficiência para a comercialização de produtos agrícolas que a cooperativa pode proporcionar é uma importante ferramenta para a organização. Sharma \& Savihi (2014) examinaram o impacto das cooperativas de leite no processamento e no marketing em Mandi, Índia. Como resultado obteve-se que o impacto foi o desconto que pode ser gerado no preço de aquisição dos insumos e um aumento da sustentabilidade financeira do local onde a cooperativa atua. 
Nota-se, ainda, diversificação no quesito comercialização, embora a maior dos entrevistados tenha respondido cooperativas, parte expressiva respondeu sobre outros canais. Os produtores familiares têm conseguido progressivamente investir no circuito da venda direta e das feiras agropecuárias, mediante formas de coordenação e de organização específicas e de certo modo inovadoras, construídas em torno das relações sociais de reciprocidade associadas à comercialização (SABOURIN et al., 2014).

Gastal et al. (2014) analisaram o acesso da agricultura familiar do município de Unaí, MG, a dois mercados - a feira de agricultura familiar e a venda de produtos para o Programa Nacional de Alimentação Escolar (PNAE). Como resultado obteve-se que é necessário que essas novas oportunidades constituam novas redes de comercialização baseados na lógica da confiança e de relações estreitas, principalmente entre agricultores e poder público.

Oliveira et al., (2010) realizaram estudo comparativo entre famílias de agricultores ecologistas do município de Ipê - RS, no que se refere à produção com base nos princípios de agroecologia. Como resultado obtiveram que na agricultura familiar a busca de autonomia social não requer necessariamente uma recusa pelos mercados, mas pode ser construída a partir de novas e estratégicas relações com estes.

\section{Participação em Curso de Gestão Rural.}

Os resultados sobre a participação em algum curso de gestão da propriedade rural podem ser visualizados na tabela 11.

Tabela 11 - Participação em algum curso de Gestão na propriedade rural

\begin{tabular}{lcc}
\hline & $\begin{array}{c}\text { Proprietários } \\
\text { rurais }\end{array}$ & Percentuais (\%) \\
\hline Nunca participei. & $\mathbf{1 2}$ & $\mathbf{5 2 , 1 7}$ \\
Participação 1 vez. & $\mathbf{3}$ & $\mathbf{1 3 , 0 4}$ \\
Participação 2 vezes. & $\mathbf{2}$ & $\mathbf{8 , 7 0}$ \\
Participação 3 vezes. & $\mathbf{2}$ & $\mathbf{8 , 7 0}$ \\
Participação 4 ou mais & $\mathbf{4}$ & \\
vezes. & & $\mathbf{1 7 , 3 9}$ \\
\hline
\end{tabular}

Fonte: Elaborado pelos autores.

Observa-se que 12 entrevistados (52,17\%) afirmam nunca terem participado de cursos voltados para esta área, o que representa uma lacuna que possa ser preenchida através de extensão rural das instituições de ensino.

Para atingir um controle eficiente, vantagens competitivas e maior produtividade, o produtor rural deve se capacitar e estar preparado para enfrentar a atividade agrícola, administrando sua propriedade através dos conhecimentos adequados. Isso facilita e otimiza as tomadas de decisões de curto prazo, pois tratam de questões que podem afetar a renda do produtor e comprometer a continuidade da prática agropecuária (HIRAKURI, 2011).

Klein et al. (2014) realizaram estudo para evidenciar o papel desempenhado pelas propriedades rurais pedagógicas na promoção da educação. Como resultado conclui-se que essas propriedades constituíam verdadeiros laboratórios de aprendizagens que contribuem sobremaneira para o processo de educação.

\section{CONCLUSÕES}

O presente estudo demonstra a situação de 23 produtores rurais entrevistados e constata que a gestão utilizada ainda pode ser melhorada de forma a ajudar na eficiência da propriedade rural.

Com um melhor gerenciamento das propriedades rurais os produtores tendem a diminuir custos e alavancar lucros, através do uso de ferramentas de controle como fluxo de caixa, classificação de custos fixos e variáveis e identificação de resultados e retornos.

Cabe ressaltar que a realização de um curso é salutar visto que $52,17 \%$ dos entrevistados nunca estiveram em um curso sobre gestão rural em Uberlândia - MG.

\section{REFERÊNCIAS BIBLIOGRÁFICAS}

BATALHA, M. O.; SILVA, A. L. Gerenciamento de sistemas agroindustriais: definições e correntes metodológicas. In: BATALHA, Mário Otávio (Coord.). Gestão Agroindustrial. 2. ed., v. 1, São Paulo: Atlas, 2001.

BRASIL, Ministério da Saúde. Secretaria de Atenção à Saúde. Atenção à saúde da pessoa idosa e envelhecimento. Área Técnica Saúde do Idoso. Brasília, 2010. 44 p.: il. (Série B. Textos Básicos de Saúde) (Série Pactos pela Saúde 2006, v. 12). Disponível em: < http://bvsms.saude.gov.br/bvs/publicacoes/atencao_saude _pessoa_idosa_envelhecimento_v12.pdf> . Acesso em 01 de ago. 2014.

BRASIL. Lei n. 11.326, de 24 de julho de 2006. Estabelece as diretrizes para a formulação de política nacional de agricultura familiar e empreendimentos familiares rurais. Diário Oficial da União, Brasília, DF, 25 jul. 2006. Disponível <http://www.planalto.gov.br/ccvil_03/_Ato20042006/2006/Lei/L11326.htm>. Acesso em: 06 fev. 2015.

BROZOVA, H; SUBRT,T; BARTOSKA, J Knowledge maps in agriculture and rural development. Agricultural Economics-Zemedelska Ekonomika. v. 54, n.11, p. 546$553,2008$.

CAMPOS, A. L.; SANTOS, N. M. B. F.; RODRIGUES, J. L. K. Gestão de recursos humanos e avaliação do desempenho por competências: análise do processo em uma empresa do vale do Paraíba. Revista ENIAC Pesquisa, v. 3, n. 1, p. $34-54,2014$.

CARVALHO, D. M.; PRÉVOT, F.; MACHADO, J. A. D. O uso da teoria da visão baseada em recursos em propriedades rurais: uma revisão sistemática da literatura. Revista de Administração, v. 49, n. 3, p. 506 - 518, 2014.

COSTA, A. H. S.; SILVA, S. S.; LIMA, V. I. A.; PORDEUS, R. V.; PEREIRA, J. O. Diagnósticos rurais participativos nas unidades de produção familiar no estado do Rio Grande do Norte através da EMATER. Revista Verde, v. 9 , n. 4 , p. $36-43,2014$ 
COSTA, N. A. O. B. Tecnologia da informação e a gestão das organizações rurais: análise sobre publicações em periódicos brasileiros. Latin American Journal of Business Management, Taubaté - SP, v. 3, n. 2, p. 159-173, 2012.

GARCIA, D. C.; FERNANDES, E. P. A importância do controle de custos na criação de gado de corte em confinamento na fazenda Sucanga. Revista da Escola de Negócios, n. 1, p. 84 - 99, 2014.

GARRIDO, L. R.; SEHNEM, S. Gestão associativa no empreendimento rural. Revista de Administração, v. 5, n. 8, p. 65-88, 2006.

GASTAL, M. L.; XAVIER, J. H. V.; ROCHA, J. C. C. G.; MENDONÇA, A. P. B.; SILVA, W. H. Construção social de mercados pela agricultura familiar em UNAI- MG: potencialidades e limitações. Cadernos de Ciência e Tecnologia, v. 31, n. 2, p. 315 - 348, 2014.

GODINHO, R. F.; SOARES, V. E.; BERTIPAGLIA, L. M. A.; DIAN, P. H. M. Gestão empresarial em sistemas de produção de leite na microrregião de São João Batista do Glória (MG). Ciencia et práxis, v. 6, n. 12, p. 39-49, 2013.

HIRAKURI, M. H - Circular técnica 88- Paraná 2011: Embrapa Soja, 2011.

IBGE - INSTITUTO BRASILEIRO DE GEOGRAFIA E ESTATÍSTICA. Censo 2010. Disponível em: < http://www.censo2010.ibge.gov.br/resultados_do_censo20 10.php>. Acesso em 02 de Jan. 2015.

KLEIN, A. L.; SOUZA, M.; TROIAN, A. Educação ambiental em propriedades rurais pedagógicas: um mundo de experiências, sabores e saberes. Revista do Programa de Pós-graduação em educação ambiental da FURG - RS, v. 31, n. 1, p. 41-59, 2014.

MAGRO, C. B. D.; VOGT, M.; CUNHA, L. C.; DEGENHART, L.; ROSA, F. S. Contabilidade rural e de custos aplicada à atividade leiteira: um estudo de caso em uma propriedade rural do Oeste de Santa Catarina. Anais do XXI Congresso Brasileiro de Custos - Natal, RN, 2014.

MARTINS, H. C.; MUYLDER, C. F.; LOPES, C. A.; FALCE, J. Os impactos da difusão tecnológica na bovinocultura leiteira: um estudo dos integrantes da cadeia agroindustrial do leite em um município em Minas Gerais. Ciência Rural, Santa Maria, v. 44, n. 6, p. 1141 1146, 2014.

MINAYO, M. C. de S. (org.). Pesquisa social: teoria, método e criatividade. $4^{\mathrm{a}}$ ed. Petrópolis: Vozes, 1994.

NAGAOKA, M. P. T.; ENSSLIN, S. R.; ENSSLIN, L.; NAGAOKA, A. Gestão de uma fazenda experimental utilizando o Balanced Scorecard. Latin American Journal of Business Management, v. 2, n. 2, p. 3-19, 2011.
NANTES, José Flávio Diniz; SCARPELLI, Moacir. Gestão da produção rural no agronegócio. In: BATALHA, Mário Otávio (Coord.). Gestão agoindustrial. 2. ed., v. 1, São Paulo: Atlas, 2001.

OLIVEIRA, D.; SILVA, M. K.; SCHNEIDER, S. A conquista da autonomia através dos mercados: como pequenos agricultores familiares mudaram sua forma de produzir e comercializar a produção em Ipê (RS). Revista Antropolítica, Niterói, n. 28, p. 249-282, 2010.

PINTO, M. A. B. A.; FRANCO, C. Custos de produção de gado bovino em regime de confinamento: análise do método de custeio por absorção em uma propriedade rural de Tangará da Serra - MT. Revista UNEMAT de Contabilidade, v. 3, n. 5, p. 401-418, 2014.

REVISTA GLEBA. Informativo Técnico da CNA. Produção cresce 7\% e Brasil poderá exportar no ano 2000. Brasília, v. 43, n. 144, p. 6-7, out. 1997.

SABOURIN, E.; THOMAS, S.; EGRET, L.; AVILA, M. L. Inovação social na comercialização dos produtos orgânicos e agroecológicos da agricultura familiar no Distrito Federal. Sustentabilidade em debate, v. 5, n. 3, p. 98-119, 2014.

SANTOS, G. J. dos; MARION, J. C.; SEGATTI, S. Administração de custos na agropecuária. 3 ed., São Paulo: Atlas, 2002.

SERVIÇO DE APOIO ÀS MICRO E PEQUENAS EMPRESAS DE MINAS GERAIS - SEBRAE - MG. Diagnóstico da indústria de laticínios do estado de Minas Gerais. Belo Horizonte, 1997.

SHARMA, K. D.; SAHIVI, P. Milk Cooperatives Innovative institutions of milk processing and marketing. Agricultural economics reserch review, v. 27, p. 213-214, 2014.

SILVA, U. M. Extensão Universitária: a interação do conhecimento na Semana do Fazendeiro. Viçosa: UFV. 199 p. 1995. Dissertação de Mestrado.

TSCHARNTKE, T.; ClOUGH, Y.; WANGER, T. C.; JACKSON, L.; MORTZE, I.; PERFECTO, I.; VANDERMEER, J.; WHITBREAD, A. Global food security, biodiversity conservation and the future of agricultural intensificantion. Biological Conservation, v. $151,2012$.

VASCONCElOS, A. C. P.; SIlVA, T. S.; SILVA; A. A. Projeto de extensão universitária: assistência técnica sobre a fertilidade e manejo do solo de propriedades rurais de Uberlândia (MG) e entorno. Revista em extensão, Uberlândia, v. 10, n. 2, p. 55-63, 2011. 